\title{
Infección por Helicobacter pylori en pacientes sintomáticos con patología gastroduodenal benigna. Análisis de 5.664 pacientes
}

\author{
JUAN PABLO ORTEGA ${ }^{1}$, ALBERTO ESPINO$^{2}$, \\ ALFONSO CALVO B. ${ }^{3}$, PATRICIA VERDUGO ${ }^{3}$, MARTHA PRUYAS $^{4}$, \\ EVA NILSEN $^{4}$, LUIS VILLARROEL ${ }^{5}$, OSLANDO PADILLA ${ }^{5}$, \\ ARNOLDO RIQUELME ${ }^{2}$, ANTONIO ROLLÁN $^{6}$
}

\section{Helicobacter pylori infection in symptomatic patients with benign gastroduodenal diseases. Analysis of 5.664 cases}

Infection with Helicobacter pylori (H. pylori) is highly prevalent in Chile, but there are no systematic studies in patients with upper gastrointestinal symptoms. Aim: To determine the prevalence of $H$. pylori infection, according to age, gender and endoscopic pathology in a large sample of patients. Methods: We studied 7,893 symptomatic patients submitted to upper gastrointestinal endoscopy between July 1996 and December 2003 in the context of a screening program of gastric cancer in a high risk population. H. pylori infection was determined by rapid urease test (RUT) in antral mucosa. We excluded 158 patients with gastric cancer (2\%) and 2,071 patients without RUT. Results: We included 5,664 patients, mean age 50.7 \pm 13.9 years, women $72.1 \%$. Endoscopic diagnoses were normal in $59.3 \%$, erosive esophagitis in 20\%, gastric ulcer (GU) in $8.1 \%$, duodenal ulcer (DU) in $6.4 \%$, and erosive gastropathy in $6.2 \%$. RUT was positive in $78 \%$ of patients. After adjusting for age and sex and with respect to patients with normal endoscopy, frequency of $H$. pylori infection was $86.6 \%$ in DU (OR 2.1, 95\% CI 1.5-2.8, $p<0.001) ; 81.4 \%$ in GU (OR 1.8, 95\% CI 1.4-2.4; $p<0.001$ ); 79.9\% in erosive gastropathy (OR 1.4, $95 \%$ CI 1.03-1.8; $p=0.03$ ) and 77.4\% in erosive esophagitis (OR 1.1, 95\% CI: 0.91.3; $p=N S)$. The probability of $H$. pylori infection decreased significantly with age, more markedly in men with normal endoscopy. Conclusions: Prevalence of H. pylori infection is very high in symptomatic Chilean patients and even higher in those with gastroduodenal ulcer or erosions, while in patients with erosive esophagitis is similar to those with normal endoscopy. The frequency of infection decreases with age, probably as a consequence of rising frequency of gastric mucosal atrophy.

(Rev Med Chile 2010; 138: 529-535).

Key words: Helicobacter pylori, Epidemiology, Peptic ulcer, Gastroscopy. 
$\mathrm{H}$ ace más de 20 años que Marshall y Warren cultivaron en mucosa gástrica humana una bacteria Gram negativa, conocida actualmente como Helicobacter pylori (H. pylori) y demostraron su rol etiopatogénico en la úlcera péptica gastroduodenal ${ }^{1,2}$. Su trabajo generó una revolución en la comprensión y tratamiento de patologías digestivas tan importantes como úlcera péptica y cáncer gástrico (CG), por lo que fueron premiados con el Premio Nobel de Fisiología y Medicina 2005.

La infección por H. Pylori afecta aproximadamente a $50 \%$ de la población mundial ${ }^{3}$. Se adquiere mayoritariamente en la infancia y presenta una baja frecuencia relativa $(20-40 \%)$ en países desarrollados y una alta frecuencia (hasta 90\%) en países en desarrollo. En Chile, en población adulta asintomática alcanza 73\% (IC 95\% 70-76\%) ${ }^{4}$. Se asocia a una gastritis de intensidad variable, generalmente asintomática. Casi toda la información publicada sobre esta infección proviene de países desarrollados y es posible que muchos de los conceptos y conductas derivados de este conocimiento, no puedan simplemente extrapolarse a los países en vías de desarrollo.

Hasta $15 \%$ de los sujetos infectados desarrollarán una úlcera péptica en el curso de su vida, encontrándose la bacteria en más de $90 \%$ de las úlceras duodenales y en 70\% de las úlceras gástri$\operatorname{cas}^{5-7}$. La erradicación de la infección se asocia a la curación de la úlcera en la mayoría de los casos y disminuye su recurrencia ${ }^{8}$. Por otro lado, una pequeña proporción de los infectados desarrollará un CG, generalmente precedido por una gastritis crónica atrófica.

La infección por $H$. pylori se ha relacionado con otras patologías digestivas de alta frecuencia, como el reflujo gastroesofágico (RGE) y la dispepsia funcional (DF). La información disponible es contradictoria, pero sugiere que existe una correlación inversa entre la frecuencia de RGE y la de $H$. pylori, más evidente en países con alta prevalencia de infección ${ }^{9-11}$. Respecto a la DF, una de las causas más frecuentes de consulta ambulatoria por síntomas digestivos, el rol etiológico del $H$. pylori es incierto. La mayoría de los estudios publicados provienen de países con baja frecuencia de infección y no muestran diferencias significativas entre dispépticos y controles ${ }^{11-13}$, aunque la última revisión sistemática de la Cochrane sugiere que la erradicación de $H$. pylori se asocia a una pequeña, pero significativa mejor respuesta sintomática respecto a placebo ${ }^{14}$. Una vez más, existe escasa información de áreas geográficas con alta frecuencia de infección ${ }^{15}$.

El objetivo principal del presente estudio fue determinar la prevalencia de infección por $H$. pylori en pacientes con síntomas digestivos con indicación clínica de endoscopia digestiva alta y correlacionar la frecuencia de la infección con las patologías más frecuentemente diagnosticadas.

\section{Pacientes y Método}

Se realizó un estudio prospectivo de pacientes que consultaron por dolor o molestia abdominal alta mayor a dos semanas de evolución, que fueron derivados al Centro de Referencia de Salud de La Florida, comuna urbana de Santiago de Chile con una alta mortalidad por cáncer gástrico ${ }^{4}$ y que fueron sometidos a endoscopia digestiva alta y búsqueda de $H$. pylori, en el contexto de un programa de pesquisa precoz de cáncer gástrico ${ }^{16}$.

Se emplearon endoscopios de fibra óptica Olympus, modelos GIF E y P3, con técnica estándar, luego de consentimiento informado. Todos los procedimientos fueron realizados por el mismo endoscopista (AC), bajo sedación moderada con midazolam i.v. La determinación de $H$. pylori se realizó mediante test de ureasa rápido (CLOtest ${ }^{\circledR}$, Kimberly-Clark $)^{17}$ en una biopsia obtenida del antro proximal.

Se incluyeron pacientes estudiados entre julio de 1996 y noviembre de 2003. Se excluyeron los que habían usado recientemente antibióticos $(<1$ mes), antagonistas de receptores $\mathrm{H} 2 \mathrm{o}$ inhibidores de la bomba de protones ( $<2$ semanas) y aquellos en los que no se realizó test de ureasa, por falta de disponibilidad del test al momento de la endoscopia. También se excluyeron los pacientes con CG, objeto de un reporte previo ${ }^{16}$. Sólo se consideró la endoscopia inicial de cada paciente.

De acuerdo a los hallazgos endoscópicos los pacientes fueron clasificados en las siguientes categorías: 1) Normal; 2) Esofagitis erosiva; 3) Gastropatía erosiva; 4) Úlcera gástrica; 5) Úlcera duodenal. Los pacientes con más de una lesión fueron categorizados de acuerdo a la de mayor gravedad (U. gástrica $>$ U. duodenal $>$ Esofagitis erosiva $>$ Gastropatía erosiva). Alteraciones inespecíficas de la mucosa gástrica como congestión, 
eritema, o disminución aparente de los pliegues, generalmente diagnosticados como "gastritis", o aquellos con alteraciones inespecíficas de la mucosa esofágica, diagnosticada a menudo como "esofagitis no erosiva", fueron consideradas normales.

Análisis estadístico: Se utilizó test chi-cuadrado para comparar la presencia de $H$. pylori entre distintos grupos. Para cuantificar el riesgo de $H$. pylori en las distintas patologías se calcularon Odds Ratio (OR) crudos con intervalo de confianza de 95\%. Para determinar variables asociadas en forma conjunta con el resultado del test de ureasa se ajustaron modelos de regresión logística binaria. Estos modelos permitieron obtener OR del riesgo de infección por $H$. pylori para las distintas patologías ajustados por edad y sexo. Para mostrar la relación entre la edad y la prevalencia de $H$. pylori se representó el logit de la probabilidad $[\operatorname{logit}(\mathrm{p})=$ $\ln (\mathrm{p} /(1-\mathrm{p}))]$ según diagnóstico y sexo, que tiene la ventaja de ser monótona creciente en p y lineal en los regresores. Se utilizó el programa SPSS 15.0 para Windows, considerándose significativo valor $\mathrm{p}<0,05$.

\section{Resultados}

Durante el período de estudio, se realizaron 9.616 endoscopias en 7.893 pacientes. Se excluyeron 2.229 por los criterios previamente descritos, de los cuales 158 (2\%) correspondieron a pacientes con CG. Un total de 5.664 pacientes fueron finalmente incluidos.

La Tabla 1 muestra las características demográficas de la muestra. La edad promedio fue de 50,7 años, con rango entre 14 y 90 años. El 72,1\% fueron mujeres. El test de ureasa fue positivo en $78 \%$ de los casos, sin diferencias significativa por sexo $(78,5 \%$ y $77,8 \%$ en hombres y mujeres, respetivamente; $\mathrm{p}=\mathrm{NS}$ ).

La Tabla 2 muestra la frecuencia de los diagnósticos endoscópicos. La endoscopia fue normal en $59,3 \%$ de los pacientes. La esofagitis erosiva estuvo presente en $20 \%$, constituyéndose en la patología más frecuente de la muestra. La úlcera gástrica fue más frecuente en pacientes de mayor edad (74,4\% de los casos en mayores de 50 años), mientras la úlcera duodenal se distribuyó a lo largo de todo el espectro etario (25,5\% en menores de 40 años y $34,3 \%$ en mayores de 60 ).
La Tabla 3 muestra que la distribución de los hallazgos endoscópicos fue significativamente distinta al comparar ambos sexos $(\mathrm{p}<0,0001)$. Una endoscopia normal fue más frecuente en mujeres, mientras todos los hallazgos patológicos, excepto la gastropatía erosiva, fueron más frecuente en hombres.

La Tabla 4 muestra la asociación entre infección por $H$. pylori y patología endoscópica, en comparación con la población con endoscopia normal. Se observa una asociación significativa entre infección por $H$. pylori y gastropatía erosiva, úlcera gástrica y úlcera duodenal, que es más marcada en relación con esta última. En la muestra estudiada, casi $90 \%$ de los pacientes con úlcera duodenal y más de $80 \%$ de los ulcerosos gástricos portaban la bacteria. No hubo asociación, en cambio, entre infección por $H$. pylori y esofagitis erosiva.

En la Figura 1 se muestra la frecuencia de $H$. pylori por grupo etario, para el grupo con endoscopia normal y para aquellos con úlcera gástrica o duodenal. A partir de la cuarta década se aprecia una tendencia a la disminución, que parece más

Tabla 1. Características demográficas

\begin{tabular}{|ll|}
\hline Pacientes incluidos $-\mathrm{n}$ & 5.664 \\
$\begin{array}{l}\text { Edad, años } \\
\text { promedio } \pm \mathrm{DE} \text { (rango) }\end{array}$ & $50,7 \pm 13,9(14-90)$ \\
$\begin{array}{l}\text { Sexo }-\mathrm{H} / \mathrm{M} \\
(\%)\end{array}$ & $1.522 / 4.142(26,9 / 72,1)$ \\
\hline $\begin{array}{l}\text { Infectados por } \mathrm{H} . \text { pylori* } \\
\mathrm{n}(\%)\end{array}$ & $4.417(78 \%)$ \\
\hline $\begin{array}{l}\text { Infección por } \mathrm{H} . \text { pylori por } \\
\text { sexo }(\%)-\mathrm{H} / \mathrm{M}\end{array}$ & $78,5 / 77,8 \%$ \\
\hline
\end{tabular}

*Test de ureasa rápido $(+)$ en mucosa antral.

Tabla 2. Distribución de frecuencia de diagnósticos endoscópicos

\begin{tabular}{|lcc|}
\hline Diagnóstico & $\mathbf{n}$ & \% \\
\hline Normal & 3.357 & 59,3 \\
\hline Esofagitis erosiva & 1.131 & 20 \\
\hline Úlcera gástrica & 462 & 8,1 \\
\hline Úlcera duodenal & 365 & 6,4 \\
\hline Gastropatía erosiva & 349 & 6,2 \\
\hline
\end{tabular}


evidente en los pacientes con endoscopia normal y más tardía en los pacientes con úlcera duodenal.

La Figura 2 muestra un análisis multivariado (logit de probabilidades) del riesgo de infección por H. pylori en función de la edad, sexo y diagnós- tico endoscópico. En esta muestra la probabilidad de infección disminuye significativamente con la edad y esta disminución es significativamente mayor en hombres, especialmente en aquellos con endoscopia normal.

Tabla 3. Diagnóstico endoscópico por sexo

\begin{tabular}{|lccc|}
\hline & $\begin{array}{c}\text { Masculino } \\
\mathbf{n ~ ( \% )}\end{array}$ & $\begin{array}{c}\text { Femenino } \\
\mathbf{n ~ ( \% )}\end{array}$ & OR (IC 95\%) \\
\hline Normal & $751(49,3)$ & $2.606(62,9)$ & $0,57(0,51-0,65)^{*}$ \\
\hline Esofagitis erosiva & $361(23,7)$ & $770(18,6)$ & $1,36(1,18-1,57)^{*}$ \\
\hline Gastropatía erosiva & $82(5,4)$ & $267(6,4)$ & $0,83(0,64-1,07)$ \\
Úlcera gástrica & $182(12,0)$ & $280(6,8)$ & $1,87(1,53-2,29)^{*}$ \\
Úlcera duodenal & $146(9,6)$ & $219(5,3)$ & $1,90(1,52-2,38)^{*}$ \\
\hline Población total & $1.522(100)$ & $4.142(100)$ & \\
\hline
\end{tabular}

${ }^{*} p<0,05$.

Tabla 4. Frecuencia de infección por H. pylori por diagnóstico endoscópico

\begin{tabular}{|lccccc|}
\hline Diagnóstico & $\begin{array}{c}\text { Test Ureasa (+) } \\
\mathbf{n}(\%)\end{array}$ & $\mathbf{P}$ & $\begin{array}{c}\text { OR crudo } \\
\text { (IC) }\end{array}$ & OR ajustado* (IC) & P \\
\hline Normal & $2.571(76,6)$ & - & 1 & & \\
Esofagitis & $875(77,4)$ & NS & $1,05(0,89-1,23)$ & $1,1(0,9-1,3)$ & NS \\
Gastropatía & $279(79,9)$ & NS & $1,22(0,93-1,60)$ & $1,4(1,03-1,8)$ & 0,03 \\
Úlcera gástrica & $376(81,4)$ & 0,02 & $1,34(1,04-1,71)$ & $1,8(1,4-2,4)$ & $<0,001$ \\
Úlcera duodenal & $316(86,6)$ & $<0,001$ & $1,97(1,44-2,69)$ & $2,1(1,5-2,8)$ & $<0,001$ \\
\hline
\end{tabular}

*Ajuste por edad y sexo mediante regresión logística binaria.

Figura 1. Infección por H. pylori en función de la edad y grupo diagnóstico.

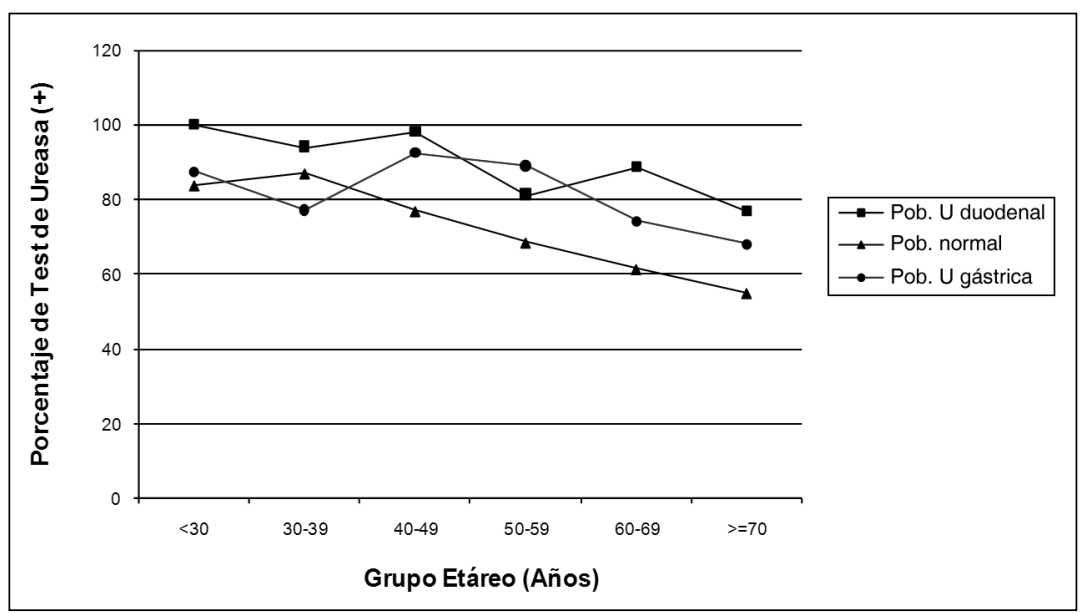




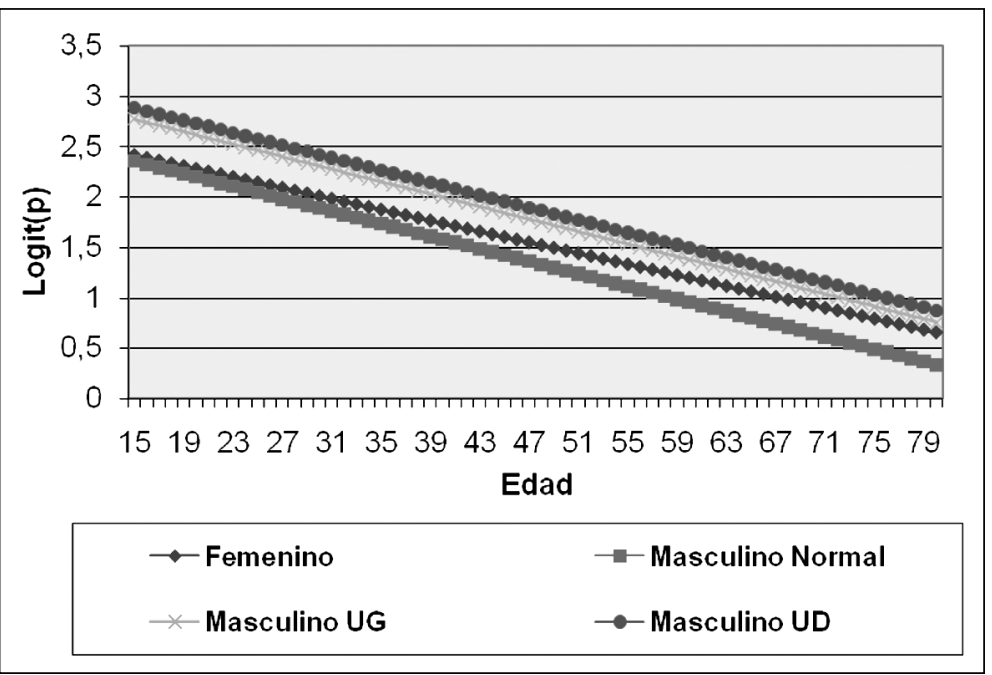

Figura 2. Infección por $H$. pylori en función de la edad, sexo y grupo diagnóstico. Análisis multivariado (Logit de Probabilidad).

\section{Discusión}

Este estudio comprende una gran serie de casos de pacientes sintomáticos provenientes de un área geográfica caracterizada por una alta frecuencia de infección por $H$. pylori y alta mortalidad por CG, condiciones que lo distinguen de la mayor parte de las publicaciones relacionadas con el tema. Las conclusiones son probablemente aplicables a poblaciones que compartan estas características, hasta ahora subrepresentadas en la literatura disponible. Es importante destacar que en Chile existe una evidente heterogeneidad respecto al riesgo de CG, siendo más frecuente en comunas y población de menor nivel socio-económico, hacia las cuales deben enfocarse predominantemente las conclusiones de este estudio.

El tamaño muestral nos permitió demostrar correlaciones y diferencias imposibles de analizar en muestras de menor tamaño. La categorización de los hallazgos endoscópicos fue exigente, sin considerar como patológicos hallazgos con alto componente subjetivo ("gastritis") y dudosa representación sintomática. Desde esta perspectiva, la demostración de patología en $40 \%$ de los pacientes sugiere que la endoscopia estuvo en general bien indicada, especialmente en el caso de los hombres. La evidente sobrerepresentación de las mujeres ( $70 \%$ de la muestra) y la significativa menor frecuencia de hallazgos patológicos en ellas ( $37,1 \%$ vs $50,7 \%$ en los hombres; $p<0,001$ ), sugieren que, en esta población, probablemente el sexo determina diferencias en los patrones de consulta médica, aunque el estudio no fue diseñado para entregar información causal al respecto, de modo que no contamos con un registro detallado de la sintomatología que motivó la indicación de endoscopia.

La esofagitis erosiva constituyó la principal patología detectada, afectando a $20 \%$ de los pacientes. Si consideramos que la mayoría de los pacientes con RGE no tiene esofagitis endoscópica ${ }^{18}$, la proporción de pacientes con RGE debe ser aún mayor. La coexistencia de una alta frecuencia de RGE con una alta frecuencia de $H$. pylori y el que la prevalencia de esta última haya sido similar en los pacientes con y sin esofagitis sugieren que, al menos en esta población, la infección por HP no parece tener un efecto protector relevante respecto al RGE.

La úlcera gastroduodenal estuvo presente en $15 \%$ de los pacientes y se asoció significativamente a la presencia de $H$. pylori. El que esta asociación sea significativa aun en una población con tan alta frecuencia basal de infección, apoya el rol etiológico de H. pylori en la úlcera péptica. A diferencia de la tendencia que se observa en países desarrollados, en los que la proporción de úlceras no asociadas a $H$. pylori va en aumento ${ }^{19}$, la infección fue casi universal en los pacientes ulcerosos y sugiere que la erradicación empírica de $H$. pylori en pacientes con úlcera gastroduodenal es probablemente costo-efectiva en esta población.

La significativa asociación entre infección por H. pylori y gastropatía erosiva es esperable dado que, junto con los antiinflamatorios no 
esteroidales, es considerada el principal factor etiológico para esta patología. A diferencia de la esofagitis y la úlcera, no existe un correlato claro entre la alteración anatómica y las manifestaciones clínicas. Es imposible saber en cuantos de estos pacientes (la mayor parte probablemente con síntomas inespecíficos y por lo tanto calificables de "dispépticos") los síntomas estaban relacionados con las erosiones gástricas. Sólo la erradicación de la infección y un posterior seguimiento clínico y endoscópico pudiera contestar esta pregunta. La erradicación de $H$. pylori ha sido sugerida como la terapia de elección para los pacientes dispépticos en la mayor parte de las guías clínicas de países desarrollados ${ }^{20}$, aun cuando la mayor parte de los pacientes persistirá sintomático luego del tratamiento ${ }^{14}$. En nuestro entorno de infección casi universal la indicación de erradicación de $H$. pylori a todos los pacientes dispépticos tiene múltiples implicancias potenciales, que demandan estudios de costo-efectividad aún no disponibles. En el intertanto, erradicar al menos a aquellos con gastropatía erosiva parece una conducta razonable, sobre todo considerando la reconocida asociación entre erosiones antrales y úlcera péptica ${ }^{21}$ y el hecho de que hasta $8 \%$ de los pacientes dispépticos con endoscopia normal desarrollan una úlcera durante el seguimiento ${ }^{22}$. Es plausible suponer que el grupo con erosiones antrales concentre especialmente a los ulcerosos no reconocidos, que son precisamente los que más se benefician de la erradicación de H. pylori. Esta conducta requiere ser validada con estudios específicamente diseñados.

El tamaño de la muestra permitió realizar análisis de subgrupos. La frecuencia de infección por $H$. pylori muestra una tendencia descendente con la edad, que es mayor en hombres y en aquellos con endoscopia normal. Este hallazgo reproduce lo observado previamente en un estudio serológico de población asintomática ${ }^{4}$. Es probable que la progresiva instalación de atrofia gástrica $(A G)$ determine un ambiente hostil para la sobrevivencia de $H$. pylori y probablemente sea una característica común en países con alta prevalencia de $H$. pylori y CG, aunque no tenemos conocimiento de que haya sido descrita previamente. Esto contrasta con lo observado en países con baja prevalencia de H. pylori, donde la frecuencia de la infección aumenta en la población de mayor edad ${ }^{23}$, lo que se ha atribuido a un efecto de cohorte determinado por la disminución progresiva en el riesgo de infección en la infancia. La Figura 1 muestra que la caída en la frecuencia de $H$. pylori es menor y más tardía en los pacientes con úlcera duodenal, que tienen menor riesgo de $\mathrm{AG}$ y $\mathrm{CG}^{24}$, lo que parece apoyar esta interpretación, aunque la ausencia de histología impide confirmar esta hipótesis. En estudio epidemiológico previo comprobamos un significativo ascenso en la frecuencia de $A G$ (determinada por serología) a contar de los 60 años, que coincidió inversamente con la caída en la frecuencia de infección por $\mathrm{HP}^{25}$. Esta observación tiene implicancias prácticas: para identificar la población con riesgo de CG en nuestro medio es necesario detectar especialmente el grupo con AG y $H$. pylori (-), ya que concentra el mayor riesgo de CG. El diagnóstico simultáneo de ambas condiciones puede realizarse en forma no invasiva mediante la determinación serológica de pepsinógeno y anticuerpos contra $H$. pylori en población asintomática, y mediante histología en pacientes sintomáticos con indicación de endoscopia. Probablemente en todo paciente sometido a una endoscopia alta debiera realizarse un test de ureasa para detectar y eventualmente tratar la infección por H. pylori, pero estos datos permiten sugerir que en hombres y mujeres mayores de 40 y 50 años, respectivamente (10 años antes del quiebre respectivo en la frecuencia de CG en población chilena) debiera realizarse además biopsias gástricas para detectar AG. Nuevamente, determinar el protocolo de biopsias más costo efectivo requiere estudios específicos.

En resumen, comprobamos una alta frecuencia de infección por $H$. pylori en pacientes sintomáticos digestivos con endoscopia normal, que es aun mayor en pacientes con úlcera gastroduodenal o gastropatía erosiva, pero no en aquellos con esofagitis por RGE, la patología benigna del tubo digestivo alto más frecuente. La infección casi universal permite sugerir la erradicación empírica (aun sin infección demostrada) como una estrategia razonable en pacientes ulcerosos en nuestra población. La disminución en la frecuencia de $H$. pylori demostrada en los pacientes de mayor edad confirma lo observado previamente en población asintomática ${ }^{4,25}$, sugiere que pudiera tratarse de una característica particular de poblaciones con alta frecuencia de $H$. pylori y CG y plantea la conveniencia de agregar la histología al estudio endoscópico en los pacientes dispépticos que concentran el riesgo de CG. 
Agradecimientos: Queremos agradecer la revisión del manuscrito y consejos de nuestro querido Profesor Dr. Vicente Valdivieso Dávila. Departamento Gastroenterología, Facultad de Medicina, Pontificia Universidad Católica de Chile.

\section{Referencias}

1. Marshall BJ, Warren JR. Unidentified curved bacilli in the stomach of patients with gastritis and peptic ulceration. Lancet 1984; 1: 1311-5.

2. Johannes GK, Arnoud HM, Ernst JK. Pathogenesis of Helicobacter pylori Infection. Clin Microbiol Rev 2006; 19: 449-90.

3. Valenzuela EJ. Helicobacter pylori: La revolución bacteriológica. Rev Med Chile 1999; 127: 891-3.

4. Ferreccio C, Rollán A, Harris PR, Serrano C, Gederlini A, Margozzini P, et al. Gastric cancer is related to early Helicobacter pylori infection in a high-prevalence country. Cancer Epidemiol Biomarkers Prev 2007; 16: 662-7.

5. Rathbone BJ, Wyatt JL, Heatley RV. Campylobacter pyloridis: a new factor in peptic ulcer disease? Gut 1986; 27 : 635-41.

6. Tygat G, Dixon MF. Overview. In: Northfield TC, Mendalí M, Goggin PM, editors. Helicobacter pylori infection. Pathophysiology, Epidemiology and Management. London: Kluwer Academic Publishers 1993; p. 75-87.

7. Mitani K, Tatsut M, Iishi, Yano H, Uedo N, Iseki K, Narahara H. Helicobacter pylori infection as a risk factor for gastric ulceration. Hepatogastroenterology 2004; 51: 309-12.

8. Rollán A, Giancaspero R, Fuster F, Acevedo C, Figueroa $\mathrm{C}$, Hola $\mathrm{K}$, et al. The long-term reinfection rate and the course of duodenal ulcer disease after eradication of Helicobacter pylori in a developing country. Am J Gastroenterol 2000; 95: 50-6.

9. Weston AP, Badr AS, Topalovski, Cherian R, Dixon A, Hassanein RS. Prospective evaluation of the prevalence of gastric Helicobacter pylori infection in patients with GERD, Barrett's esophagous, Barrett's dysplasia, and Barrett's adenocarcinoma. Am J Gastroenterol 2000; 95: 387-94.

10. Raghunath AS, Hungin AP, Wooff D, Childs S. Systematic review: the effect of Helicobacter pylori and its eradication on gastro-oesophageal reflux disease in patients with duodenal ulcers or reflux oesophagitis. Aliment Pharmacol Ther 2004; 20: 733-44.

11. Abe Y, Ohara S, Koike T, Sekine H, Iijima K, Kawamura $\mathrm{M}$, et al. The prevalence of Helicobacter pylori infection and the status of gastric acid secretion in patients with Barrett's esophagus in Japan. Am J Gastroenterol 2004; 99: 1213-21.

12. Talley NJ, Vakil N, Ballard D, Fennery MB. Absence of benefit of eradicating Helicobacter pylori in patients with nonulcer dyspepsia. N Engl Med 1999; 341: 1106-11.

13. Laine L, Shoenfeld P, Fennetry MB. Therapy for H. pylori in patients with nonulcer dyspepsia. A meta-analysis of randomized controlled trials. Ann Intern Med 2001; 134: 361-9.

14. Moayyedi P, Soo S, Deeks J, Delaney B, Harris A, Innes $\mathrm{M}$, et al Eradication of Helicobacter pylori for non-ulcer dyspepsia. Cochrane Database Syst Rev 2006; CD002096. Disponible en: www.cochrane.org [Consultado el 20 de septiembre de 2009].

15. Gwee KA, Leyan T, Wong RK, Ho KY, Sutedja DS, Yeoh KG. The response of Asian patients with functional dyspepsia to eradication of Helicobacter pylori infection. Eur J Gastroenterol Hepatol 2009; 21: 417-24.

16. Calvo A, Pruyas M, Nielsen E, Verdugo P. Pesquisa poblacional de cáncer gástrico en pacientes sintomáticos digestivos, período 1996-2000. Rev Med Chile 2001; 129 : 749-55.

17. Viiala $\mathrm{CH}$, Windsor H, Forbes $\mathrm{G}$, Chairman SO, Mollison LC. Evaluation of a new formulation CLOtest. J Gastroenterol Hepatol 2002; 17: 127-30.

18. Ronkainen J, Aro P, Storskrubb T, Johanson SE, Lind T, Bolling-Sternevald E, et al. High prevalence of gastroesophageal refl ux symptoms and esophagitis with or without symptoms in the general adult Swedish population: a Kalixanda study report. Scand J Gastroenterol 2005; 40: 275-85.

19. Leong RW. Differences in peptic ulcer between the East and the West. Gastroenterol Clin North Am 2009; 38:363-79.

20. Nimish V, Francis M. Eradication Therapy for Helicobacter pylori. Gastroenterology 2007; 133: 985-1001.

21. Zhang CH, Yamada N, Wu YL, Wen M, Matsuhisa T, Matsukura N. Helicobacter pylori infection, glandular atrophy and intestinal metaplasia in superficial gastritis, gastric erosion, erosive gastritis, gastric ulcer and early gastric cancer. World J Gastroenterol 2005; 11: 791-6.

22. Hsu PI, Lai KH, Lo GH, Tseng HH, Lo CC, Chen HC, et al. Risk factors for ulcer development in patients with non-ulcer dyspepsia: a prospective two year follow up study of 209 patients. Gut 2002; 51:15-20.

23. Dooley CP, Cohen H, Fitzgibbons PL, Bauer M, Aplleman MD, Pérez-Pérez GI, et al. Prevalence of Helicobacter pylori infection and histologic gastritis in asymptomatic persons. N Engl J Med 321; 1562-6.

24. Hansson LE, Nyrén O, Hsing AW, Bergström R, Josefsso $\mathrm{S}$, Chow WH, et al. The risk of stomach cancer in patients with gastric or duodenal ulcer disease. N Engl J Med 1996; 335: 242-9.

25. Rollan A, Ferreccio C, Gederlini A, Serrano A, Torres J, Harris P. Non-invasive diagnosis of gastric mucosal atrophy in an asymptomatic population with high prevalence of gastric cancer. World J Gastroenterol 2006; 12 : 7172-8. 